\section{Le signe de Brunet-Guedj}

\section{Brunet-Guedj's sign}

\section{Y. Daniel · F. Moulis $\cdot$ A. Rocheteau $\cdot$ P. Mardegan}

Reçu le 16 février 2011 ; accepté le 8 mars 2011

(C) SFMU et Springer-Verlag France 2011

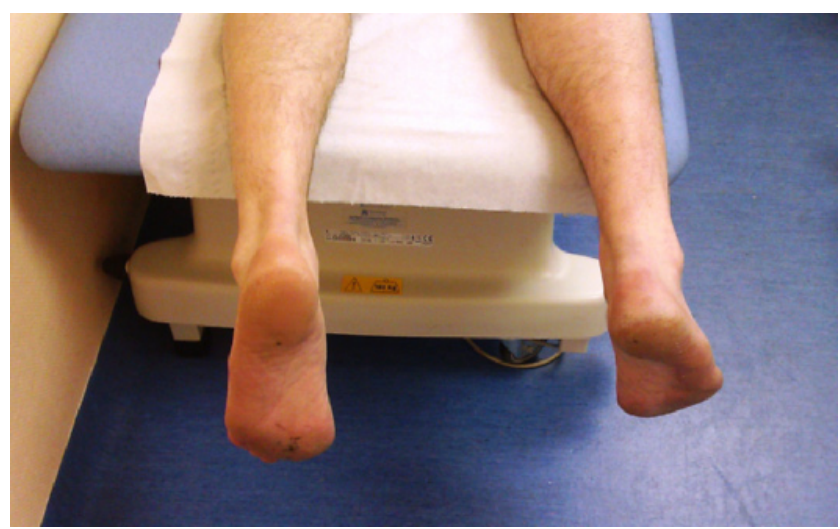

Un patient de 42 ans se présente pour une impotence fonctionnelle majeure de la cheville droite, douloureuse, suite à un mouvement de pivot, lors d'un match de handball. On ne note aucun critère d'Ottawa. En revanche, l'inspection montre en décubitus ventral, une asymétrie de la position des pieds. C'est le signe de Brunet-Guedj.

La perte de l'équinisme naturel du pied, appelée dans les pays francophones « signe de Brunet-Guedj » est quasi pathognomononique de la rupture complète du tendon achilléen. Son équivalent anglo-saxon est le "Matles test ». Sa sensibilité et sa spécificité sont respectivement évaluées à $0,88(\mathrm{IC} 95 \%=[0,79-0,95])$ et à 0,86 (IC $95 \%=[0,67-$ $0,96])[1]$.

Comme le rappelle l'American Academy of Orthopaedic Surgeons dans ses recommandations, son association à un autre signe clinique de rupture du tendon calcanéen signe le diagnostic [2]. Trois autres éléments sont donc à rechercher : la recherche d'une déhiscence à la palpation du tendon, le déficit de la flexion plantaire du pied et le signe de Thompson (absence de mouvement du pied, à la pression des gastrocnémiens). Ces deux derniers signes étaient présents chez notre patient. La déhiscence peut, en effet, être comblée par l'hématome, particulièrement si le patient est vu tardivement.

Conflit d'intérêt : les auteurs ne déclarent aucun conflit d'intérêt.

\section{Références}

1. Maffulli N (1998) The clinical diagnosis of subcutaneous tear of the Achilles tendon. A prospective study in 174 patients. Am J Sports Med 26: 266-70

2. Chiodo CP, Glazebrook M, Bluman EM, et al (2010) Diagnosis and treatment of acute Achilles tendon rupture. J Am Acad Orthop Surg 18:503-10

\footnotetext{
Y. Daniel $(\bowtie)$

Service médical du $17^{\mathrm{e}}$ régiment du génie parachutiste, Montauban, France

e-mail : yadaniel@hotmail.fr 